\title{
Análisis de la red de diagnóstico de malaria, 2008
}

\author{
Aguilar $\mathrm{M}^{1}$, Mendoza NM ${ }^{1}$ \\ 1. Instituto Nacional de Salud, Subdirección Red Nacional de Laboratorios \\ Grupo de Parasitología \\ Correspondencia:
}

Recibido: 15-06-09 / Aceptado: 22-11-09

\begin{abstract}
Resumen
Desde 1995, año en el que se conforma la red de diagnóstico de malaria a nivel nacional, hasta la fecha, el grupo de Parasitología-Red Nacional de Diagnóstico del Instituto Nacional de Salud ha implementado el Programa de Malaria dentro de la Red Nacional de Laboratorios, desarrollando diversas actividades con el fin de fortalecer el diagnóstico y tratamiento oportuno de esta enfermedad en el país. La red de diagnóstico de malaria está distribuida en 989 municipios y constituida por 1.640 laboratorios clínicos y 1.308 puestos de microscopia, datos que revelan un aumento del $13,4 \%$, en la cobertura de la red municipal, una disminución del 3,5\% en el número de laboratorios clínicos y un aumento del I I,8\% en los puestos de microscopía con relación al año 2007.

El presente trabajo tuvo como objetivo evaluar la cobertura de la red y la calidad del diagnóstico de malaria durante el año 2008. Estos resultados no cubren la información de los departamentos de Magdalena y Casanare. Se diagnosticaron 387.993 muestras, de las cuales 61.304 fueron positivas. Para P.vivax 40.937, para P. falciparum 20.0 13, 353 para asociación y I para P. malariae. En cuanto a la evaluación externa directa del desempeño departamental fue: concordancia: 92,7\%, la cobertura del 100\% y la participación del 96.9\%. El 60,6\% de laboratorios obtuvieron una concordancia superior al 95\%. La evaluación externa indirecta de desempeño fue: concordancia (+): 99,3\%; concordancia (-): 100\%, índice kappa: 0,99, IKE: 0,95, participación: 96,9\%. Para la evaluación externa directa del desempeño, a nivel municipal, se obtuvieron los siguientes datos: concordancia $=84,9 \%$, cobertura: 40,7\%, y para la evaluación externa indirecta: concordancia (+): 95,3\% y concordancia (-): 98, 1 \%, índice kappa: 0.94, IKE: 0,79, cobertura: 89,2\%.
\end{abstract}

Palabras clave: anopheles, enfermedad parasitaria, malaria, protozoo.

\section{Abstract \\ Analysis of malaria diagnostic network, 2008}

The national diagnosis of malaria network is founded in 1995. Since then, the group of parasitology, the National Diagnostic Network of the National Institute of Health has implemented the Malaria Program within the National Laboratory Network by developing various activities in order to strengthen the diagnosis and treatment of this disease in the country. The malaria diagnostic network exists in 989 municipalities and consists of I,640 clinical laboratories and I,308 microscopy posts, these data show an increase of $13.4 \%$ in the municipal network coverage, a decrease of $3.5 \%$ the number of clinical 
laboratories and an increase of $11.8 \%$ in microscopy posts with respect to the year 2007.

This study aimed to evaluate the network coverage and quality of the malaria diagnosis in 2008. These results do not cover the information from the departments of Magdalena and Casanare. 387,993 samples were diagnosed, out of which 61,304 were positive. For P. vivax 40,937; for P. falciparum 20,0 I 3; 353 for association and I for P. malariae. As for the direct external evaluation of the departmental performance was: agreement: $92.7 \%$, coverage of $100 \%$ and $96.9 \%$ participation. $60.6 \%$ of laboratories obtained a concordance above $95 \%$. The indirect external evaluation of performance was: concordance $(+): 99.3 \%$ concordance (-): 100\%, kappa 0.99, IKE: 0.95, Participation: 96.9\%. To direct external evaluation of performance, at the municipal level, we obtained the following data: concordance $=84.9 \%$ coverage: 40.7\%, and for the indirect external evaluation was: correlation (+): 95.3\% and concordance (-): $98.1 \%$, kappa: 0.94, IKE: 0.79, coverage: 89.2\%.

Keywords: anopheles, malaria, parasitic disease, protozoan.

\section{Introducción}

La malaria es una enfermedad producida por protozoos que se transmiten por la picadura del mosquito Anopheles infectado. Cada ańo causa la muerte de más dos millones de personas en el mundo y se presenta en unos 400 millones de casos, en los 103 países donde se ha establecido su presencia. Ha sido erradicada de Europa, Rusia y Estados Unidos. A pesar de los enormes esfuerzos realizados para controlarla ha reaparecido en muchas zonas tropicales. Además, existen cada vez más problemas de resistencia farmacológica del parasito y de los vectores a los insecticidas. Al igual que hace muchos siglos, esta enfermedad trae no sólo dificultades a quienes la padecen, también a sus familias a los sistemas de salud y a la sociedad en general (1).

En Colombia la malaria es una patología de alto poder epidémico, es endémica en una gran parte del territorio nacional localizado por debajo de los 1.600 m.s.n.m. (2) y aproximadamente 25 millones de personas habitan en zonas de riesgo. Si bien la mortalidad por malaria ha disminuido en Colombia, en los últimos años se registra un incremento en la incidencia de la enfermedad, especialmente por $P$. falciparum. Esta situación constituye un evento cuya vigilancia, prevención y control revisten especial interés en salud pública, que requiere un diagnóstico oportuno y adecuado.
La implementación de políticas que garanticen acceso a un tratamiento adecuado se fundamenta necesariamente en la existencia de un sistema de atención que ofrezca con oportunidad acceso a un diagnóstico confiable. La calidad en la preparación y lectura de la gota gruesa en el diagnóstico de malaria requiere de la existencia de procedimientos y herramientas que permitan promover y monitorear la calidad del mismo con base en la estructura de la red de laboratorios (3).

El presente trabajo muestra la situación de la red de diagnóstico en malaria en el año 2008. A través del Laboratorio de Parasitología- Red Nacional de Laboratorios (RNL), el Instituto Nacional de Salud (INS)se obtiene información del control de calidad de los laboratorios a nivel municipal y departamental (4).

\section{Materiales y métodos}

Con el fin de evaluar la cobertura de la red y la calidad del diagnóstico de malaria, se realizó un estudio de tipo descriptivo retrospectivo. Las fuentes de información fueron los condensados anuales de actividades procedentes de los 32 laboratorios departamentales de salud, de la secretaria de salud de Bogotá, de los laboratorios y puestos de microscopía en el nivel municipal y de los informes de control de calidad realizados por el Instituto Nacional de Salud 
a los entes territoriales durante el año 2008. También se realizó la evaluación externa del desempeño directa (EEDD) y la evaluación externa indirecta del desempeño (EEID). Para la presente evaluación no enviaron información los departamentos de Casanare y Magdalena.

Parámetros evaluados: índice kappa (IK) (5), concordancia $(\mathrm{C})$, media aritmética y frecuencia relativa (5) y promedio ponderado para el análisis de los datos.

Procedimiento para EEID: se solicita al laboratorio participante 60 láminas de un periodo epidemiológico determinado, 30 positivas y 30 negativas, y a partir de su revisión se obtienen indicadores de concordancia, de ser inferior a la muestra se revisa la totalidad del material.

Procedimiento para EEDD: se envían láminas problema a los laboratorios participantes y se evalúa la concordancia del diagnóstico tanto de especie como de formas parasitarias observadas.

Para la evaluación departamental se analizaron los resultados de las actividades realizadas por el Laboratorio Nacional de Referencia (LNR) a los Laboratorios Departamentales de Salud Pública (LDSP) (6), haciendo énfasis en la EEDD y en la EEID. Para la evaluación a nivel municipal se procesaron los condensados de actividades del programa de malaria reportadas al INS por los 33 laboratorios del nivel departamental.

\section{Resultados}

\section{Conformación de la red de diagnóstico de malaria}

Está distribuida en 989 municipios y constituida por 1640 laboratorios clínicos y 1308 puestos de microscopia, datos que revelan un aumento del $13,4 \%$ en la cobertura de la red municipal, una disminución del 3,5\% en el número de laboratorios clínicos y un aumento del 11,8\% en los puestos de microscopía con relación al año 2007, Tabla 1.

\section{Reporte de diagnósticos}

En el año 2008 los departamentos que mayor número de casos reportaron fueron: Antioquia, Chocó, Nariño, Guaviare y Córdoba. La relación de casos de P. vivax y $P$. falciparum fue de $2: 1$. Se diagnosticaron 387.993 muestras, fueron positivas 61.304, para P. vivax 40.937, para P. falciparum 20.013, 353 para asociación y 1 para $P$. malariae, Tabla 2.

\section{Evaluación externa directa e indirecta del desempeño}

Nivel departamental. En la EEDD del nivel departamental, el 60,6\% de los laboratorios obtuvieron una concordancia superior al 95,0\% (92,7\%), aunque se presentó una disminución de $2,7 \%$ en la concordancia promedio. La cobertura fue del 100\% y

Tabla 1. Conformación de la Red de Diagnóstico del Programa de Malaria - RNL.

\begin{tabular}{cccc|}
\hline \multicolumn{4}{c|}{ CONFORMACIÓN RED DE DIAGNÓSTICO } \\
\hline AÑO & NÚMERO DE & NÚMERO DE & NÚMERO DE PUESTOS \\
& MUNICIPIOS & LABORATORIOS & DE MICROSCOPIA \\
2007 & 872 & 1700 & 1170 \\
2008 & 989 & 1640 & 1308 \\
\hline
\end{tabular}

Tabla 2. Reporte de diagnósticos de malaria.

\begin{tabular}{ccccccc}
\hline AÑO & $\begin{array}{c}\text { TOTAL MUESTRAS } \\
\text { EXAMINADAS }\end{array}$ & $\begin{array}{c}\text { TOTAL } \\
\text { POSITIVA }\end{array}$ & $\begin{array}{c}\text { Plasmodium } \\
\text { vivax }\end{array}$ & $\begin{array}{c}\text { Plasmodium } \\
\text { falciparum }\end{array}$ & $\begin{array}{c}\text { INFECCIÓN } \\
\text { MIXTA }\end{array}$ & $\begin{array}{c}\text { Plasmodium } \\
\text { malariae }\end{array}$ \\
$\mathbf{2 0 0 7}$ & 564755 & 125272 & 70753 & 53852 & 10 & 657 \\
$\mathbf{2 0 0 8}$ & 387993 & 61304 & 40937 & 20013 & 353 & 1 \\
\hline
\end{tabular}


la participación del $96.9 \%$. El 60,6\% de laboratorios obtuvieron una concordancia superior al 95\%. EEID: C (+): 99,3\%; C (-): 100\%, IK: 0,99, IKE: 0,95, participación: $96,9 \%$. Estos se muestran en la Tabla 3.

Nivel municipal. La cobertura de la EEDD a nivel municipal aumentó en un 6\%. La EEID se realizó a 2948 puestos de diagnóstico entre laboratorios clínicos y puestos de microscopía; la cobertura aumentó en un $21,7 \%$ con respecto al año 2007. EEDD: C: 84,9\%, cobertura: 40,7\%. EEID: C (+): 95,3\% у C (-): 98,1\%, IK: 0.94, IKE: 0,79, cobertura: $89,2 \%$, Tabla 4 .

\section{Discusión}

El programa de malaria cuenta con un buen cubrimiento en las actividades de la red de laboratorio a nivel nacional y el LNR proporciona apoyo a todos y cada unos de los laboratorios que pertenecen a la red, independientemente de su rendimiento o participación (7). De acuerdo con la concordancia y el IK de la EEID a nivel departamental y municipal, se considera que la calidad el diagnóstico de malaria es adecuada. Sin embargo, es importante que a nivel departamental como municipal, fortalecer la concordancia en el diagnóstico en la actividad de la EEDD y la cobertura tanto de la EEDD como de la EEID a nivel municipal.

La participación departamental en la actividad de EEDD fue de $100 \%$, resultado que refleja un mayor interés en las actividades de la red de diagnóstico de malaria por parte de los LDSP. Debido a que el mejoramiento del acceso del diagnóstico de malaria, mediante la ampliación de la red de microscopía, ha sido un lineamiento nacional que se ha trabajado en los últimos años, es importante el aumento de la cobertura tanto en los municipios como en el número de puestos de microscopía que realizan el diagnóstico.

\section{Referencias}

1. Mendoza NM, Nicholls RS, Olano VA, Cortés LJ. Manejo integral de malaria. Instituto Nacional de Salud. 2000.

2. Garcia, Marisol.; Mendoza Nohora Marcela. Evaluación Del programa de diagnóstico de malaria en La Red de laboratorios de Colombia, 1997 - 1999. Biomédica, Junio 22: 002. 2002. Bogotá. 123-132.

3. World Health Organization. Consultado el: 21/09/09. Disponible en: http://www.wpro.who.int/internet/resources.ashx/MVP/malaria_light_microscopy.pdf.

4. Protocolo de vigilancia para malaria. Evento de vigilancia y control. Primer semestre 2007.

5. Guía para la implementación de un sistema de gestión de calidad en el diagnóstico microscópico de malaria: Estandarización de procedimientos y herramientas sobre el control de calidad y la evaluación externa del desempeño en las redes de laboratorio. Borrador de trabajo. Propuesta de un grupo técnico, Caracas, Venezuela, julio de 2004

6. Organización Panamericana de la Salud. Consultado el: 20/09/09. Disponible en: www.paho.org/English/AD/DPC/CD/ravreda6quality-mgt.doc

7. Condensado de actividades LDSP y SDS de Bogotá 2007 y 2008.

Tabla 3. Evaluación Externa Directa e Indirecta del Desempeño Nivel Departamental EYALUACIÓN EXTERNA DIRECTA E INDIRECTA DEL DESEMPEÑO NIVEL DEPARTAMENTAL

\begin{tabular}{|c|c|c|c|c|c|c|c|c|}
\hline \multirow[t]{2}{*}{ AÑO } & \multicolumn{3}{|c|}{ EEDD } & \multicolumn{5}{|c|}{ EEID } \\
\hline & CONCORDANCIA & COBERTURA & PARTICIPACIÓN & $\begin{array}{c}\text { CONCORDANCIA } \\
(+)\end{array}$ & $\begin{array}{c}\text { CONCORDANCIA } \\
(-)\end{array}$ & $\begin{array}{c}\text { INDICE } \\
\text { KAPPA } \\
\text { (IK) }\end{array}$ & $\begin{array}{c}\text { IK DE } \\
\text { ESPECIE }\end{array}$ & PARTICIPACIÓN \\
\hline 2007 & 95,4 & $100 \%$ & $95,40 \%$ & 99,5 & 99,5 & 0,99 & 0,96 & 96,9 \\
\hline 2008 & $92,7 \%$ & $100 \%$ & $96,6 \%$ & $99,3 \%$ & $100 \%$ & 0,99 & 0,95 & 96,9 \\
\hline
\end{tabular}

Tabla 4. Evaluación Externa Directa e Indirecta del Desempeño Nivel Municipal EVALUACION EXTERNA DIRECTA E INDIRECTA DEL DESEMPEÑO NIVEL MUNICIPAL

\begin{tabular}{|c|c|c|c|c|c|c|c|}
\hline \multirow[t]{2}{*}{ AÑO } & \multicolumn{2}{|c|}{ EEDD } & \multicolumn{5}{|c|}{ EEID } \\
\hline & CONCORDANCIA & COBERTURA & $\begin{array}{l}\text { CONCORDANCIA } \\
(+)\end{array}$ & $\begin{array}{c}\text { CONCORDANCIA } \\
(-)\end{array}$ & $\begin{array}{c}\text { INDICE } \\
\text { KAPPA } \\
\text { (IK) }\end{array}$ & $\begin{array}{l}\text { IK DE } \\
\text { ESPECIE }\end{array}$ & COBERTURA \\
\hline 2007 & $78,80 \%$ & $34,7 \%$ & 94,7 & 97,2 & 0,93 & 0,88 & $67,5 \%$ \\
\hline 2008 & $84,9 \%$ & $40,7 \%$ & $95,3 \%$ & $98 \%$ & 0,94 & 0,79 & $89,2 \%$ \\
\hline
\end{tabular}

